\title{
The Application of an Improved Fuzzy Petri Net Model in Fault Diagnosis of Breakers
}

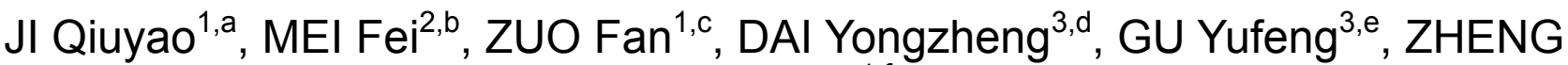 \\ Jianyong ${ }^{1, f}$
}

${ }^{1}$ School of Electrical Engineering, Southeast University, Nanjing, 210096;

${ }^{2}$ College of Energy and Electrical Engineering, Hohai University, Nanjing 211100, China;

3 Jiangsu NariTurbostar Electric Co.Ltd, Jiangsu Taizhou, 225300

a617496576@qq.com, ${ }^{\mathrm{b}} 19763452 @ q q . c o m,{ }^{\mathrm{C}} 850527240 @ q q . c o m,{ }^{d}$ daiyongzheng@sgepri.sgcc.co m.cn, ${ }^{e}$ guyufeng@sgepri.sgcc.com.cn, ${ }^{\mathrm{f}} 101003914 @$ seu.edu.cn

Keywords: Fuzzy petri net; Rough set theory; Fault diagnosis

\begin{abstract}
Fast development of smart grid has made it essential to promote the intelligent level of electrical equipment. As circuit breakers is an important kind of control and protection equipment in power system, its on-line monitoring and fault diagnosis are significant parts of electrical equipment intelligence. Traditional Petri net is used to establish graph-based models to detect faults while the application of fuzzy Petri net makes decision of complex faults more accurate.In this paper, fuzzy Petri net is combined with rough set theory which is utilized to simplify the diagnosis rules. The combination of the two algorithms can not only improve diagnosis efficiency quickly but also meet the demands of on-line fault diagnosis much better. The effectiveness of theproposed method is proved by satisfactory results of field experiments.
\end{abstract}

\section{Introduction}

High voltage breakers occupy an important place in national grid security. Large amounts of work have been done in the field of high voltage breaker's on-line state monitoring and smart fault $\operatorname{diagnosis}^{[1]}$. The smart fault diagnosis contains three steps including the collection of information, the extraction of rules and the diagnosis of objects' state, of which the extraction of rules is the core of smart diagnosis ${ }^{[2]}$. The breaker's fault diagnosis is a typical artificial intelligent problem. Common artificial intelligent algorithms include expert system, neural network, data fusion, D-S evidence theory ${ }^{[3]}$ and so on.

Paper [4] applies fuzzy petri net in the electrical grid fault diagnosis which realizes a distinct and visual expression of diagnosis rules with uncertainty of grid taken into consideration. Paper [5]makes use of variable precision rough set decision tables to eliminate redundant information in breaker's on-line monitor data, laying a solid foundation for the extraction of diagnosis rules. Paper [6] combines rough set theory and petri net in the fault diagnosis of breakers, but the characteristics selected remain to be improved. On the basis of the work done, a fuzzy petri net model based on attribute importance is proposed in this paper. With the appliance of rough set theory, the decision tables can be discretized and reducted so that the redundant information can be eliminated. The combination of the two methods is quite complementary and improves the rapidity and precision of fault diagnosis efficiently.

\section{Improved fuzzy petri net model}

Fuzzy petri net model.Proposed by German mathematician A.C.Petri in 1962, Petri net is mathematical model using net figures which is currently widely used In a petri net model, the input and output are both called places. The rule of mapping from the input to the output is known as the changes. And places are connected with changes by directed arcs to transport knowledge to Petri net.

Petri net can be represented by a five-tuple array $\mathrm{PN}=<\mathrm{P}, \mathrm{T}, \mathrm{K}, \mathrm{I}, \mathrm{O}>$, where $\mathrm{P}=\left\{\mathrm{p}_{1}, \mathrm{p}_{2}, \mathrm{p}_{3}, \ldots, \mathrm{p}_{\mathrm{n}}\right\}$ is 
the finite set of places; $T=\left\{\mathrm{t}_{1}, \mathrm{t}_{2}, \mathrm{t}_{3}, \ldots, \mathrm{t}_{\mathrm{m}}\right\}$ is the finite set of changes; $\mathrm{K}=\left\{\mathrm{k}_{1}, \mathrm{k}_{2}, \mathrm{k}_{3}, \ldots, \mathrm{k}_{\mathrm{n}}\right\}$ is the finite set of token, representing the states of places; I is $\mathrm{m}^{*} \mathrm{n}$ order matrix, representing the set of all the directed arcs from places to changes. When it comes to case $\mathrm{P} \rightarrow \mathrm{T}$, elements in I are 1 , otherwise they become $0 . \mathrm{O}$ is $\mathrm{m}^{*} \mathrm{n}$ order output matrix, representing the set of all the directed arcs from changes to places. When it comes to case $\mathrm{T} \rightarrow \mathrm{P}$, elements in $\mathrm{O}$ are 1 , otherwise they become 0 .

Graphic of Petri net is a directed graph containing two types of nodes, where nodes of places are represented by hollow circles "O" and nodes of changes are represented by short long strings "|" and reasoning relations are represented by directed arcs. A simple Petri net model is shown in Fig.1.

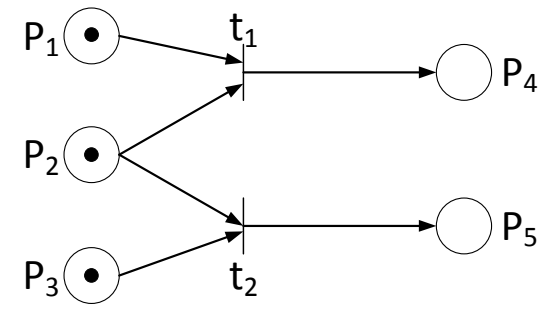

Fig. 1 a Simple Petri net model

In order to consider influence of incompleteness and uncertainty on object information, in fuzzy Petri net (FPN), the concept of probability and confidence is introduced on the basis of Petri net. A six-tuple array $\mathrm{FPN}=<\mathrm{P}, \mathrm{T}, \mathrm{I}, \mathrm{O}, \theta, \mathrm{U}>$ can be utilized to represent FPN, where the former token set $K$ is replaced by state vector $\theta=\left[\theta_{1}, \theta_{2}, \theta_{3}, \ldots, \theta_{n}\right],(\theta \in[0,1])$ representing probability of existence of places; the new-added confidence matrix

$$
U=\operatorname{diag}\left(u_{1}, u_{2}, u_{3}, \cdots, u_{m}\right)
$$

represents confidence of changes; the rest concepts remain the same and graphic of FPN is the same as the one of Petri net.

The reasoning method utilized in this paper is based on AI MYCIN. Let $\theta^{0}$ be the initial state. The forward reasoning can be conducted according to formula(2):

$$
\theta^{k+1}=\theta^{k} \oplus\left[(\mathrm{O} \bullet U) \otimes \overline{\left(\mathrm{I}^{T} \otimes \overline{\theta^{k}}\right)}\right]
$$

Calculationsymbols of which are shown as following:

$\oplus: \mathrm{A} \oplus \mathrm{B}=\mathrm{C}, \mathrm{A}, \quad \mathrm{B}, \quad \mathrm{C}$ are all $\mathrm{m} * \mathrm{n}$ order matrix, and $\mathrm{C}_{\mathrm{ij}}=\max \left(\mathrm{A}_{\mathrm{ij}}, \mathrm{B}_{\mathrm{ij}}\right)$;

$\otimes: A \otimes B=D, \quad \mathrm{~A}, \quad \mathrm{~B}$ and $\mathrm{D}$ are respectively $\mathrm{m}^{*} \mathrm{q}, \mathrm{q}^{*} \mathrm{n}, \mathrm{m}^{*} \mathrm{n}$ order matrixes, and $\mathrm{D}_{\mathrm{ij}}=\max _{l \leq k \leq q}\left(A_{i k} \cdot B_{k j}\right) ; \overline{\theta^{k}}: \overline{\theta^{k}}=\mathrm{l}_{\mathrm{m}}-\theta^{k}$, where $\theta^{k}$ represents the $\mathrm{m}$ dimensional vector of the $\mathrm{k}_{\mathrm{th}}$ step of reasoning state and $l_{\mathrm{m}}$ is an $\mathrm{m}$ dimensional vector with value of all elements being 1 ;

Repeat the steps of forward reasoning until $\theta^{k+1}=\theta^{k}$, and it comes to the end of reasoning. Place with the highest existence probability can be found through the final result of $\theta^{k}$.

FPN model based on rough set theory.The establishment of FPN is closely related with original information. If original information is too much, too large matrix and net size will be caused which reduces the speed and efficiency of reasoning, making FPN lose its original meaning. Therefore, rough set theory is utilized in this paper to pretreat characteristicquantities so as to improve the efficiency.

Rough set theory can divide uncertain objects without relying on propaedeutics. It can eliminate the redundant characteristic quantities and reveal underlying laws between objects. Decision table is one of the important concepts in rough set theory, which can organize chaotic information into clear and orderly knowledge structure.

Definition 1: decision table $\mathrm{K}=\langle U, A, V, \rho\rangle$ is a set of ordered quadruples containing all knowledge and decision arribute. Each relationship in the decision table is also called the law. In the formal formula, $\mathrm{W}=\left\{\mathrm{x}_{1}, \ldots, \mathrm{x}_{\mathrm{n}}\right\}$ is the set of all the object $\mathrm{x} ; \mathrm{A}=\mathrm{CUD}$ is the set of attributes, which is the description and the representation of objects; $C=\left\{F_{1}, F_{2}, \cdots, F_{n}\right\}$ is condition attribute, $\mathrm{D}=\left\{\mathrm{d}_{1}, \mathrm{~d}_{2}, \cdots, \mathrm{d}_{\mathrm{m}}\right\}$ is decision attribute, $\mathrm{C} \cap \mathrm{D}=\emptyset ; \mathrm{V}=\mathrm{U}_{\mathrm{a} \in \mathrm{A}} \mathrm{V}_{\mathrm{a}}$ is the ensemble of the range, where $V_{a}$ is the range, value of attribute; relationship $\rho: U \times A \rightarrow V$ is an information function 
indicating the corresponding object attribute values.

It's worthy to note that the simple decision table is for discrete information and data; however, during intelligent fault diagnosis of breakers, the collected characteristic contains both discrete and continuous data. It is the most important issue of applying the simple decision table to fault diagnosis of breakers that how to reasonably discrete the continuous data. Similar to the process of simple decision table, different breakpoints are of different importance to one attribute. Deleting some of the breakpoints has no effect on this attribute.

Steps of discrete algorithm based on importance of attribute are as following:

Step 1: As for continuous attribute $F=\left\{\mathrm{f}_{1}, \mathrm{f}_{2}, \mathrm{f}_{3} \ldots, \mathrm{f}_{\mathrm{n}}\right\}$, obtain $\mathrm{F}=\left\{\mathrm{f}_{1}^{*}, \mathrm{f}_{2}^{*}, \mathrm{f}_{3}^{*} \ldots, \mathrm{f}_{\mathrm{n}}^{*}\right\},\left(\mathrm{f}_{1}^{*} \leq \mathrm{f}_{2}^{*} \leq\right.$ $\left.\mathrm{f}_{3}^{*} \leq \cdots \leq \mathrm{f}_{\mathrm{n}}^{*}\right)$ in ascending order; Step 2: Calculate breakpoints set $P=\left\{\mathrm{p}_{1}, \mathrm{p}_{2}, \mathrm{p}_{3}, \ldots, \mathrm{p}_{\mathrm{n}-1}\right\}$ where $p_{i}$ is breakpoint value and $p_{i}=\left(f_{i}^{*}+f_{i+1}^{*}\right) / 2$; Step 3: Compare two adjacent attribute values of $p_{1}$ in decision table, namely $f_{1}^{*}$ and $f_{2}^{*}$, and change the value of $f_{2}^{*}$ to the one of $f_{1}^{*}$. If it does not cause conflict in decision table, then delete $\mathrm{p}_{1}$ from breakpoints set $\mathrm{P}$; otherwise keepp $\mathrm{p}_{1}$.

Combining with methods above, steps of simple decision table based on importance of attribute are as following:

Step1: Calculate and sorting relative importance of all the condition attributes; Step 2: Discrete continuous condition attributes on the basis of attribute importance in ascending order; Step 3: Obtain the minimum attribute set according to the constant classification quality law; Step 4: Obtain simple decision table by simplifying each law according to the minimum attribute set.

To further illustrate the reliability of the method, data set Iris and Glass are processed by using it. Result obtained is shown Table 1.

Tab.1 Results after processing data set Iris and Glass

\begin{tabular}{ccccc}
\hline Data set & $\begin{array}{c}\text { Number of } \\
\text { samples }\end{array}$ & $\begin{array}{c}\text { Number of condition } \\
\text { attributes before } \\
\text { simplification }\end{array}$ & $\begin{array}{c}\text { Number of } \\
\text { condition attributes } \\
\text { after simplification }\end{array}$ & $\begin{array}{c}\text { Number of } \\
\text { breakpoints }\end{array}$ \\
\hline Iris & 150 & 4 & 3 & 8 \\
Glass & 214 & 9 & 5 & 18 \\
\hline
\end{tabular}

It can be observed that decision table simplification based on attribute importance can compress the space occupied by a large number of knowledge well and eliminate the redundant condition attributes. It can also discrete continuous data while remaining the same resolution of decision table. When this method is applied to fault diagnosis of circuit breakers, it can extract effective fault diagnosis laws to make a good foundation of making rapid and accurate judgments for fault diagnosis.

\section{Fault diagnosis of high voltage circuit breakers based on improved FPN model}

Selecting characteristic quantities of fault diagnosis of HVCBs.Opening and closing coil current signal has obvious characteristics and is easy to extract. It contains a lot of information during operation process, reflecting many types of fault of breakers' control circuit. Therefore, opening and closing coil current signal can be extracted as characteristic value. Typical current wave is shown in Figure 2. It can be divided into 5 stages according to current amplitude:

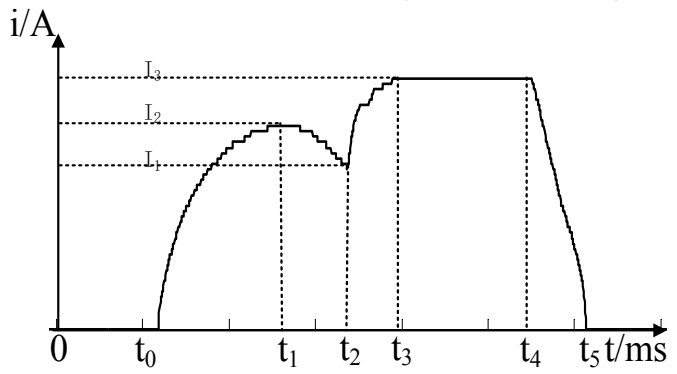

Fig.2 Typical coil current waveform when breakers close

Stage 1: $t_{0} \sim t_{1}$, at time $t_{0}$, the breaker receives closing command, the breaker's closing coil is energized but the core remained stationary. Closing current occurs and increases exponentially until 
time $t_{1}$

Stage 2: $t_{1} \sim t_{2}$, at time $t_{1}$, the core begins to move and to touch the actuator buckle. The core's motion slows down or stops when closing current declines slowly until time $t_{2}$;

Stage 3: $t_{2} \sim t_{3}$, at time $t_{2}$, closing current increases exponentially again and reaches the peak because the core stops moving due to the block of actuator buckle;

Stage 4: $t_{3} \sim t_{4}$, a continuation of last stage, current becomes steady;

Stage 5: $t_{4} \sim t_{5}$, at time $t_{4}$, the buckle of the actuator closes, the core moves again and the breaker successfully closes. Electric arc is generated between the auxiliary switch contacts, causing the voltage to increase, due to which the current increases rapidly and becomes 0 at time $t_{5}$.

According to the analysis above, condition attribute set of decision table is established by choosing breaker closing coil current value $\mathrm{I}_{1}, \mathrm{I}_{2}, \mathrm{I}_{3}$ and time parameters $\mathrm{t}_{1}, \mathrm{t}_{2}, \mathrm{t}_{3}, \mathrm{t}_{4}, \mathrm{t}_{5}$ as characteristic value for rough set in this paper. When the coil current is abnormal, there may be fault such as initial points off core jam, actuator jam, too large core closing idle stroke, too low operating voltage, as decision attribute $d_{1}, d 2, d 3, d 4$ respectively.

Process of fault diagnosis of HVCBs.The establishment of fault diagnosis model of breakers faces problems of both plenty of fault characteristic value and fault types, which is of much uncertainty and complexity. The application of FPN model can reflect the state of objects and events accurately as well as speed up fault diagnosis.

But the complexity of FPN will increase with the amount of information. So it is necessary to use effective intelligence algorithm to simplify diagnosis laws and to lower the dimension of FPN. In this paper, decision table simplification method is introduced on the basis of FPN model. Based on the basic concept of rough set, this method simplifies attributes and compress information to extract effective laws of fault diagnosis under the premise of remaining the indiscernibility relation of the decision table. It greatly simplifies fault diagnosis model and lays a good foundation for the application of FPN method. Aflowchart of breaker fault diagnosis using FPN model on the basis of rough set theory is shown in Figure 3.

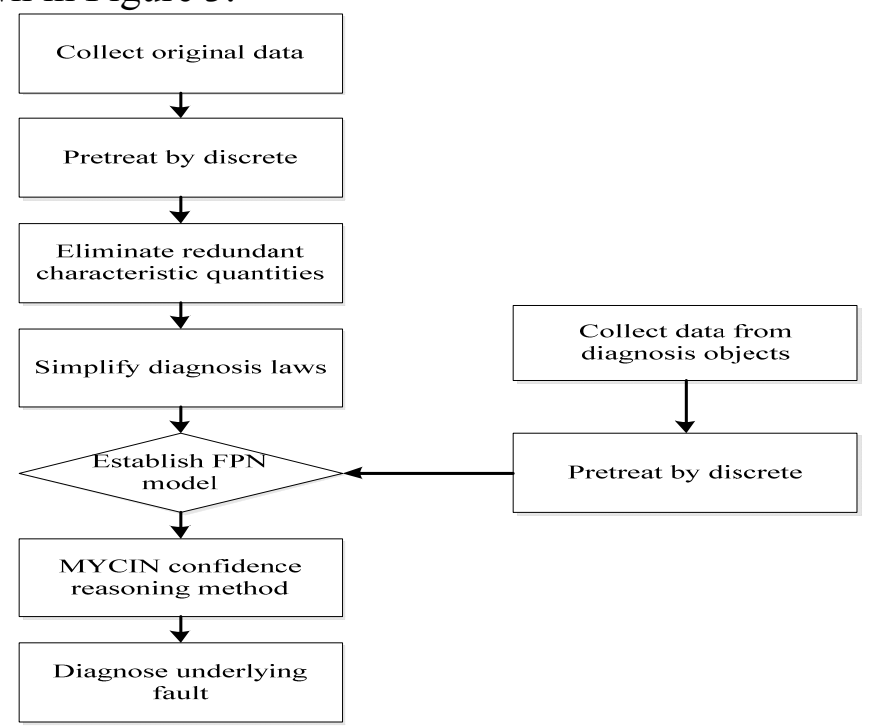

Fig.3 Flow chart of breaker fault diagnosis using FPN model based on the rough set theory

\section{Example of fault diagnosis of HVCBs}

Take a prototype of $\mathrm{ZF} 40-126 \mathrm{SF}_{6}$ breaker as an example. The diagnosis laws and net model are established by actually collected fault samples to develop online monitoring and intelligent fault diagnosis system of breaker. After establishing condition attribute set $\mathrm{C}=\left\{\mathrm{I}_{1}, \mathrm{I}_{2}, \mathrm{I}_{3}, \mathrm{t}_{1}, \mathrm{t}_{2}, \mathrm{t}_{3}, \mathrm{t}_{4}, \mathrm{t}_{5}\right\}$ and decision attribute set $\mathrm{D}=\left\{\mathrm{d}_{1}, \mathrm{~d}_{2}, \mathrm{~d}_{3}, \mathrm{~d}_{4}\right\}, 20$ groups of data are chosen to establish original decision table. The simplified decision table obtained after procession based on importance of attributes is shown in Table 2. 
Tab.2 Closing fault diagnosis rules of high-voltage breakers after reduction

\begin{tabular}{cccc}
\hline$t_{1}$ & $t_{2}$ & $t_{3}$ & $D$ \\
\hline 1 & 1 & 0 & $d_{1}$ \\
1 & 0 & 0 & $d_{2}$ \\
0 & 1 & 1 & $d_{3}$ \\
0 & 1 & 0 & $d_{3}$ \\
0 & 0 & 0 & $d_{4}$ \\
\hline
\end{tabular}

Establish FPN model as shown in Figure 4, and the corresponding input matrix is

$$
I=\left[\begin{array}{llllllllll}
1 & 0 & 1 & 0 & 0 & 1 & 0 & 0 & 0 & 0 \\
1 & 0 & 0 & 1 & 0 & 1 & 0 & 0 & 0 & 0 \\
0 & 1 & 1 & 0 & 1 & 0 & 0 & 0 & 0 & 0 \\
0 & 1 & 1 & 0 & 0 & 1 & 0 & 0 & 0 & 0 \\
0 & 1 & 0 & 1 & 0 & 1 & 0 & 0 & 0 & 0
\end{array}\right]^{T}
$$

Output matrix is

$$
O=\left[\begin{array}{llllllllll}
0 & 0 & 0 & 0 & 0 & 0 & 1 & 0 & 0 & 0 \\
0 & 0 & 0 & 0 & 0 & 0 & 0 & 1 & 0 & 0 \\
0 & 0 & 0 & 0 & 0 & 0 & 0 & 0 & 1 & 0 \\
0 & 0 & 0 & 0 & 0 & 0 & 0 & 0 & 1 & 0 \\
0 & 0 & 0 & 0 & 0 & 0 & 0 & 0 & 0 & 1
\end{array}\right]^{T}
$$

Obtain confidence matrix $U=\operatorname{diag}[0.85,0.9,0.8,0.9,0.85]$ according to references.Collect test samples of initial stages of closing core jam fault as the fault diagnosis test samples and discrete original data on the basis of importance of attribute to get the initial state $\theta^{0}=\left[\begin{array}{llllllllll}0.87 & 0.13 & 0.92 & 0.08 & 0.51 & 0.49 & 0 & 0 & 0 & 0\end{array}\right]^{\mathrm{T}}$. After reasoning based on FPN model, $\theta^{2}=$ [0.87 0.13 0.920.08 0.510.49 0.41650 .0720 .1040 .068$]^{\mathrm{T}}$ is obtainedas the result. The probability of the core jam is 0.4165 , which is far higher than other types of faults. It is consistent with the experimental conditions. And it can be concluded that the fault diagnosis method is accurate and feasible.

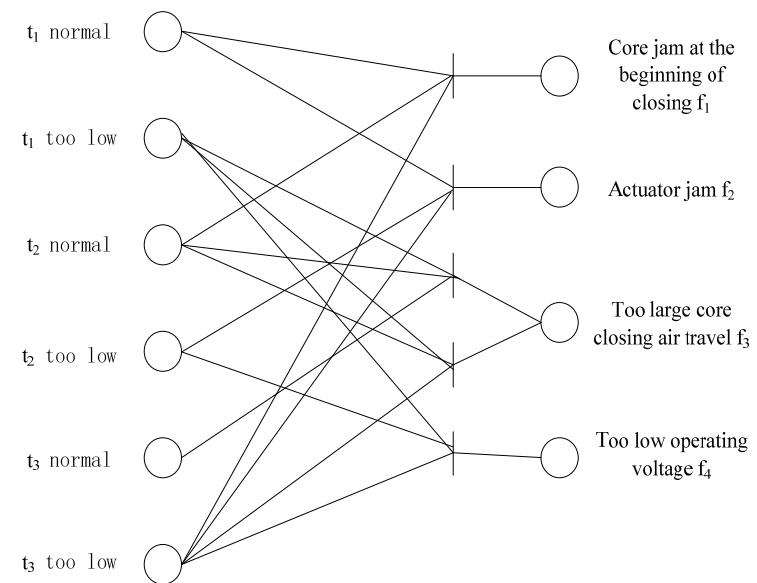

Fig.4 Closing fault diagnosis FPN model of high-voltage breakers

\section{Conclusions}

The concept of rough set theory has been applied to improve fuzzy petri net models so that redundant characteristics are eliminated and information space is compressed efficiently. Consequently, dimension of FPN reasoning matrix is decreased and the speedof reasoning is accelerated. The key timing point and value of breaker's switching coil current have been selected as characteristics, which is not only convenient to on-line monitoring but also reflects the acting situation of the entire switching process. The application of improved FPN model established during high voltage breaker's fault diagnosis can realize visual and rapid fault diagnosis and 
improve high voltage breaker's maintenanceefficiency. Experiments have proved the validity and accuracy of improved FPN models.

\section{References}

[1]Liu Zenghuan, Li Zhenzhen.Circuit breakers' fault diagnosis based on RBF neural network and expert system [J].Application of electronic technique.40(7):85-87. 2014.

[2] Jian Huang,Xiaoguang Hu,Xin Geng.An intelligent fault diagnosis method of high voltage circuit breaker based on improved EMD energy entropy and multi-class support vector machine[J]. Electric power systems research .2010 (2).

[3] Mei fei, Mei jun, Zheng jian-yong, etc.Application of particle swarm fused kfem and classification model of svm for fault diagnosis of circuit breaker [J]. Journal of Electrical Engineering.33(36): 134-141. 2013.

[4]Yang jian-wei.Research on the fuzzy petri nets based fault diagnosis for power system[D]. Chengdu: Southwest Jiaotong University. 2011.

[5]Huang xin-bo,Tao chen,Liu bin. Breaker fault diagnosis based on variable precision rough set-support vector machine [J]. Guangdong electric power.27(7): 64-67. 2014.

[6]Zhao ning,Zhao jie.Circuit breaker fault diagnosis based on tough set theory and petri net[J]. Electric power.44 (08): 9-13. 2011. 